\title{
A brief analysis of the research scheme of the cyclotron radiation from a single electron
}

\begin{abstract}
The experiments of Project 8 have been excellent, but the expected goals still difficult to achieve. So much so that some of the results at your fingertips were also missing. In view of this, the focus of this article is to clarify several easy confused concepts. Only in this way, we can reasonably explain the experimental data. The main points are as follows: 1 . The value $\mathrm{c}$ of the light speed in vacuum and a particle with zero static mass, both of which do not exist in the reality. That is to say, the so-called a photon's static mass is equal to zero but has energy, which is a paradox that confuses two different definitional domains. 2. In the reality, photons are high-speed particles generated by electromagnetic radiation. They must have the characteristics of (static) mass, energy and wave, in order to describe the main body to aim at photons from different angles. 3. After any main body comes into being electromagnetic radiation, its static mass will inevitably decrease accordingly. 4 . The charge-mass ratio of an electron is a physical constant, which is the ratio of its charge to the amount of matter, and is not affected by relativistic effects and electromagnetic radiation. 5. The uncertainty of moving electrons is caused by random electromagnetic radiations. Finally, it is pointed out that if the cyclotron frequency of a single electron is measured, and at the same time, its de Broglie wavelength or frequency can also be measured, then its static mass can be obtained. Even so, the expected goals are still difficult to achieve, because random electromagnetic radiations are always taking away continually the matter composition of the single electron being measured. However, this was precisely a result of the research obtained by Project 8, and had universality, which should be reflect on.
\end{abstract}

Keywords: electromagnetic radiation, electron, charge-mass ratio, uncertainty principle, static mass
Volume 4 Issue 2 - 2020

\author{
Jian Ding \\ Retired, Integrated Electronic Systems Lab Co. Ltd, China
}

Correspondence: Jian Ding, Male, Retired, Integrated Electronic Systems Lab Co. Ltd. Jinan 250100, China, Email jiandus@।63.com

Received: April 20, 2020 | Published: April 30, 2020
PACS: 41.60.Ap; 29.30.Dn; 03.75.-b; 29.20.-c

\section{Introduction}

In 2015, we saw a paper entitled as "Single-electron detection and spectroscopy via relativistic cyclotron radiation" in Physical Review Letters, ${ }^{1}$ which introduced the Project 8 experiment being carried out jointly by scientists from many countries. The characteristic of this experiment was that a single electron generated by the $\beta$-decay of ${ }^{83 \mathrm{~m}} \mathrm{Kr}$ was trapped in the magnetic field of $B=1(T)$, and moved along a circular orbit perpendicular to the magnetic field. At the same time, the single electron cyclotron emitted a frequency of 25 $\mathrm{GHz}$ approximately, which was able to be detected by a sensitive microwave amplifier. The scientists believed that this meant a new experimental method, which was able to use the spectrum radiated by a single electron cyclotron to determine its energy. According to the related theory, in negative beta decay, a neutron inside the nucleus was transformed into a proton, at the same time, both a beta particle and an antineutrino were released. Beta particles were electrons. The purpose of the implementation of the Project 8 by scientists was following the law of conservation of mass-energy, and attempting by accurately measuring the frequency of electromagneticradiationwhen asingleelectronwasrotatingtodetermineitsenergy. If the energy of the beta particle during decay was able to be determined, it was possible to calculate out its static mass by the kinetics formulas of Einstein's special relativity. Then compared with the difference between the static mass of a neutron and a proton, it was possible to determine the static mass of the neutrino. The so-called related theory was put forward by W.E. Pauli in 1930, in negative beta decay, an electron was released, at the same time, a neutral particle with zero static mass and $1 / 2$ spin was released. Later, the particle was called a neutrino and claimed to have been experimentally confirmed.

\section{Electromagnetic radiation will inevitably carry away matter}

If you want to discuss the Project 8 more deeply, some consensuses must be reached on the most basic concepts ${ }^{2}$ and no ambiguity should be allowed. Therefore, it is necessary to give two hints in advance to facilitate the consensuses. First, the photon moving at the value $\mathrm{c}$ of the light speed in vacuum strictly follows the rules determined by Newton's first law of motion, and its static mass can only be equal to 0 , which is the limit value of moving photons and does not exist in the reality. That is to say, the so-called a photon's static mass is equal to zero but has energy, which is a paradox that confuses two different domains. Furthermore, the photons in the reality are high-speed particles generated by electromagnetic radiation. They must have the characteristics of (static) mass, energy and wave, which are describing the main body of photons from different angles. It means that after any main body comes into being electromagnetic radiation, its static mass will inevitably decrease accordingly. ${ }^{3}$ Therefore, it is vital to correctly understand and distinction the relationship between the speed of light in vacuum and the speed of light in reality. ${ }^{4}$ So, how did the scientists of Project 8 look at the above two hints? From their experimental goal of view, it was in order to determine the mass of a neutrino. But the problem they faced was the electromagnetic radiation generated by the single electron. The energy of radiated photons was $\Delta E=\Delta m c^{2}$ and had randomness. As mentioned above, in terms of the main body of photons, the energy was sure to contain the static mass $\Delta m_{0}=\Delta m / \gamma$ representing the amount of matter, the two were collectively called 
as mass-energy. Here, $m$ was the dynamic mass when an electron's moving speed was equal to $v$, or called as the relativistic mass, which was the mass to represent inertia. And $m_{0}$ was the static mass of the single electron, also known as the rest mass, which was the mass to represent the amount of matter, while $\gamma=1 / \sqrt{1^{2}-v^{2} / c^{2}}$ was the expansion factor of Lorentz's transformation. ${ }^{3}$

This meant that the single electron to rotate each circle, its static mass $m_{0}$ would be gradually lost with the energy radiated. Moreover, the occurrence of radiation was random, and the lost static mass was also different. The static mass carried by each photon generated by these radiations was almost far higher than the static mass of a neutrino. So the mass of neutrino was highly likely to be submerged in the mass's errors lost by the radiations. If the scientists at Project 8 could be aware of this, would they still use such an experimental scheme to determine the mass of neutrino? Research on neutrinos is at the forefront of today's science. The above discussion about Project 8 is not intended to stop them, only a kind reminder. Besides, it also tells them that the experimental data of the single electron cyclotron radiation can be used to reflect on the Uncertainty principle and discuss its essence.

\section{More universal larmor theorem}

A single electron is trapped in a magnetic field of $B=1(T)$, and moving along a circular orbit of radius $r$ perpendicular to the magnetic field, and its cyclotron frequency as $f_{r}$. without considering the relativistic effects, its angular frequency

$$
\omega=2 \pi f_{r}=\frac{e}{m} B
$$

Can be obtained directly from the equation of the centripetal force $m \omega^{2} r$ and Lorentez force $e B v$. This is consistent with the Larmor Theorem.

In formula (1), the concepts related to the charge-mass ratio are once again involved. ${ }^{3}$ Initially, when defining the concept of chargemass ratio, people did not realize the existence of relativistic effects. So the charge-mass ratio $\mathrm{e} / \mathrm{m}$ at that time was defined under the non-relativistic low-speed state. The mass $\mathrm{m}$ was able to perfectly represent the inertia and the amount of matter. It seemed that there was no need to emphasize or specifically referred to the ratio of the charge to the amount of matter. But now, we must consider the relativistic effects. In terms of high-speed moving electrons, the original concept of mass in classical mechanics is divided into two, $m_{0}$ and $m$, which are represented the amount of matter and inertia respectively. This reveals that the so-called charge-mass ratio $e / \mathrm{m}$ is a variable, and the true charge-mass ratio should be $e / m_{0}$, that is, the ratio of charge to amount of matter. Only the charge-mass ratio defined by such a concept is not affected by relativistic effects and electromagnetic radiation, and still maintains the original constant value, $e / m_{0}=-1.75882 * 10^{11}(C / \mathrm{kg})$, that is charge-mass ratio of the physical constant of an electron. In this regard we have been by virtue of an electron storage ring windely in use as experimental fact, to prove that the charge e of a high-speed electron would follow along with its static mass $m_{0}$ to be lost synchronously, and its chargemass ratio $e / m_{0}$ remained unchanged. ${ }^{3}$ If you continue to regard the charge-mass ratio as $e / m$, under the premise of the given magnetic induction intensity $B$, according to the formula (2), will be possible to derive out such a wrong conclusion that the cyclotron frequency $f_{\gamma}$ of an electron in the ring is independent of its speed $v$. This conclusion is surely wrong, due to be affected by relativistic effects, the so-called charge-mass ratio $e / m$ has been no longer a constant and varied with the electron's speed $v$. It is vital whether the charge-mass ratio of electrons is a constant, because it reveals a fundamental rule of nature. In other words, an electron and the photons radiated by it are all composed of the electro-ultimate particles, ${ }^{2}$ because their textures are the same, that is, having the same charge-mass ratio. To sum up, the charge-mass ratio $e / m_{0}$ is the ratio of the amount of charge to the amount of matter, which is precisely the original intention of this physical concept defined.

Thus, substituting the mass-speed formula $m=m_{0} \gamma$ of Einstein's special relativity into the formula (1), we obtain a more universal Larmor Theorem. As follows:

$$
\omega=2 \pi f_{\gamma}=\frac{e}{m_{0}} B \sqrt{1-\frac{v^{2}}{c^{2}}}
$$

In the formula (2), its definition domain is also $0<|v|<c$, the same as the kinetics formulas of Einstein's special relativity. ${ }^{2}$

Now take the experiment of the single electron cyclotron radiation (Project 8) as an example. The formula (2) reveals that under the premise of the given magnetic induction intensity $B$, due to the charge-mass ratio $e / m_{0}$ is a constant, if the single electron's cyclotron frequency $f_{\gamma}$ can be determined by virtue of precisely measuringelectromagneticradiation, then its moving speed $v$ also will be determined accordingly. After that, according to the relative speed formula. ${ }^{3}$

$$
\beta=\frac{v}{c}=\sqrt{\frac{\left(\frac{e}{m_{0}}\right)^{2}}{\left(\frac{e}{m_{0}}\right)^{2}+\left(\frac{c}{B r}\right)^{2}}}
$$

of the cyclotron electron, its orbital radius $r$ can be also determined.

Ifwe wanttodiscussthesingleelectroncyclotronradiationexperiment (Project 8), the formulas (2) and (3) are crucial importance. Because we can use them to build a platform of non-inertial reference system, the background which relies on is the inertia reference system in the reality, defined by Newton's first law of motion. That is to say, under the premise of the given magnetic induction intensity $B$, if the radiation and other disturbances are excluded, the single electron's cyclotron frequency $f_{\gamma}$, orbital radius $r$ and moving speed $v$ are three interrelated values. Between them, as long as any one is known, the other two values can be calculated out by the formulas (2) and (3). And their respective initial values depend on the moving speed $v$ when the single electron is trapped into the magnetic field.

\section{Further discussion on the uncertainty principle}

Due to the effect of electromagnetic radiation, the static mass $m_{0}$ of each electron is not the same, but their charge-mass ratio $\mathrm{e} / \mathrm{m}_{0}$ is constant. This means that on any orbit of the platform, the static mass $m_{0}$ of each cyclotron electron can have different values as long as themoving speed $v$ follows therules determined by theformula(3). That is to say, for the electrons with the same moving speed $v$, their cyclotron frequency $f_{\gamma}$ and orbital radius $r$ must be the same, but the momentum $p$ or energy $E$ can bedifferent. Here, the charge-mass ratio $e / m_{0}$ is like a wall. On the one side of thewall, there are three interrelated values, cyclotron frequency $f_{\gamma}$, orbital radius $r$ and moving speed $v$, they are parameters to describe the location characteristics. And on the other side of the wall, there also are three interrelated values, static mass $m_{0}$, momentum $p$ and energy $E$, they are collectively called as the mass-energy.If youonlygetthevalues ofoneside,certainlywillnotbe able to calculate any value of the other side based on this. Therefore, 
measuring the nondestructive spectrum of the cyclotron electron can only determine its cyclotron frequency $f_{\gamma}$ as well as orbital radius $r$ and moving speed $v$, but cannot calculate out its momentum $p$ or energy $E$ based on them. This means that the difficulty faced by the scientists at Project 8 has been precisely the Uncertainty principle.

Thereupon, it triggers our review for the principle of uncertainty. In 1927, the German physicist Heisenberg first pointed out that the more precisely the position of some particle is determined, the less precisely its momentum can be known,and viceversa. Theprinciple canalsobe expressed as: When themomentum $p$ is determined, the speed $v$ (or position) cannot be determined; when the speed $v$ (or position) is determined, the momentum $p$ cannot be determined.

Through this platform, we can see the internal mechanism of the principle of uncertainty. The electrons with different static masses, their charge-mass ratio $e / m_{0}$ is always the same constant. When they are moving at different speeds, the momentums or energies can be the same. So the phenomenon presented is "When the momentum $p$ is determined, the speed $v$ cannot be determined." Conversely, when they are moving at the same speed $v$, the momentums or energies must be various. So the phenomenon presented is "When the speed $v$ is determined, the momentum $p$ cannot be determined."

This just proves that the static mass of each electron is different. For a low-speed electron moving in non-relativistic state, its charge, static mass and charge-mass ratio all were considered to be physical constants, which were the consensuses reached a hundred years ago. In other words, in that era of Heisenberg, physicists widely believed that an electron could not be further broken down. And now we must realize that due to the effect of electromagnetic radiation, the charge and static mass both have been nolonger the constants. As long as an energy $\Delta E=\Delta m c^{2}$ is radiated, it must be accompanied by the loss of static mass $m_{0}$ (thatis, acertain amount of matter), which respectively describe the same one event from different angles (energy and matter). In this regard, there has been no consensus. Previously, with the help of an electron storage ring as an experimental fact, we have proved that the charge e of a high-speed electron would follow along with its static mass $m_{0}$ to be lost synchronously, both of which were no longer constants, but their charge-mass ratio $e / m_{0}$ was always to remain as the physical constant. In other words, for this inference that an electron was made up of a certain number of electro-ultimate particles and both of them had the same charge-mass ratio, which has revealed out a fundamental rule of nature, we should be taken seriously. ${ }^{2}$

As the background of nature, the ether as a medium is also made up of the electro-ultimate particles, their moving speeds are the highest in the reality and second only to the c. The so-called magnetic field lines whose essence is the electro-ultimate particles and the stream of charged particles derived by there from. When an electron moving in the ether along a circle with radius $r$, there will be periodic changes in the current. It is precisely that it forces the stream of charged particles to change the density and direction near in the plane defined by the circumference. This meant that the spectrum radiated by a single electron cyclotron as discussed above could be regarded as the synthesis of two parts. One was generated by the cyclotron current of a single electron, and the other was electromagnetic radiation, both of which in essence were electromagnetic waves.

Furthermore, an electron moving in the ether medium, its wave law follows the relation formula of de Broglie's matter wave, and its momentum $p=m v=h / \lambda$ and energy $E=m v^{2}=h f$ cannot grow without limits, which must be restricted by the Planck's constant $h$. This means that if the cyclotron frequency $f_{\gamma}$ of a single electron is measured, and at the same time, its de Broglie wavelength $\lambda$ or frequency $f$ can also be measured, then its static mass $m_{0}$, as well as corresponding momentum $p$ and energy $E$ can be calculated out. But even so, the expected goals are still difficult to achieve, because random electromagnetic radiations are always taking away continually the matter composition of the single electron being measured, which should be reflect on.

An electron can be further broken down, which is the internal cause to come into being the random electromagnetic radiation. So the interactions between the ether medium and the electrons, as well as the Lorentz force, are the external cause to come into being the random electromagnetic radiation. The whole process of electromagnetic radiation follows the law of conservation of mass-energy, that is, the partial binding energy inside the electron is converted into the kinetic energy in the form of photon radiation. ${ }^{5}$

When an electron is rotating, the charge will not follow along with its static mass to be lost synchronously if there is no electromagnetic radiation, both of them will always remain near their respective values of the physical constants. In other words, the so-called uncertainty will not exist. So the uncertainty of moving electrons is caused by random electromagnetic radiations.

In the following, on the platform determined by the formulas (2) and (3), we use some experimental data of single electron cyclotron radiation, and temporarily avoid other interference factors, and only aim at random electromagnetic radiation to moderately calculate and discuss.

\section{Reasonably to explain the experimental data of project 8}

Figure 1 is derived from the paper of the above Physical Review Letters. ${ }^{1}$ In order to further clarify the viewpoint of this article, the moderate additions and deletions have been made on the premise of respecting the original data. The single electron moved in a magnetic field of $B=1(T)$, with the left vertical axes respectively denoted the cyclotron frequency $f_{\gamma}$ and the moving speed $v$, and the right vertical axis denoted the orbital radius $r$, and the horizontal axis denoted the change in time. Both theory and experiment have shown that an electron to do circular motion can emit electromagnetic radiation continuously and randomly. So the electron's charge $e$ would follow along with its static mass $m_{0}$ to be lost synchronously, and show as its energy $E$ to be decreased gradually, that is, to present convergence phenomena. If just simply judge according to the formulas (2) and (3), you will find that the reason for the decrease in energy $E$ is the loss of the static mass $m_{0}$, but this should not change its three interrelated values, that is, cyclotron frequency $f_{\gamma}$, orbital radius $r$ and moving speed $v$. Well, the cyclotron frequency $f_{\gamma}$ shown in Figure 1 was increased, which must be that the random electromagnetic radiation had changed its moving speed $v$.

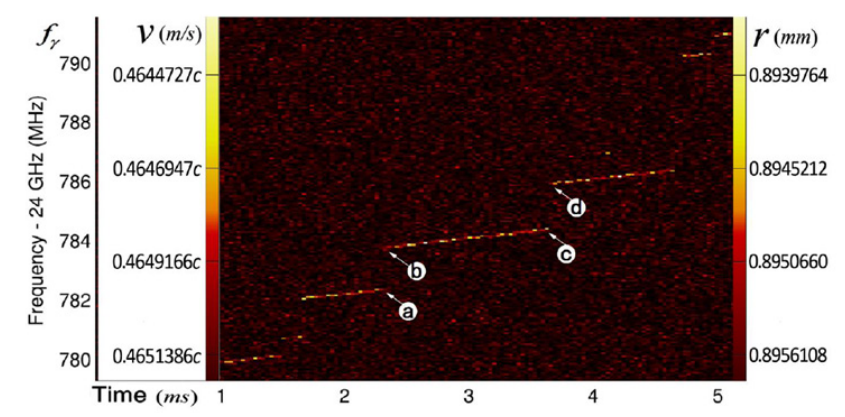

Figure I The change law of the single electron's position features in $B=I(T)$ magnetic field with time. 
For convenience of explanation in Figure 1, four points (a, b, c, d) are specifically marked in accordance with the single electron's cyclotron frequency $f_{\gamma}$. By substituting the cyclotron frequency $f_{\gamma}$ of every one of the points respectively into formula (2), the corresponding moving speed $v$ can be obtained. Similarly, the moving speed ${ }^{v}$ of every one of the points is substituted respectively into the formula (3), and the corresponding orbit radius $r$ can be obtained. See Table 1. It has been calculated in Table 1 that the single electron's moving speed $v=0.465 c$. So, the light cone of the photon radiations was centered on the tangent of the cyclotron orbit, and along the direction of the single electron's moving speed $v$, its divergence half-angle $\theta_{1 / 2} \approx 1 / \gamma$ was larger, close to $70^{\circ}$. That was to say, the impulses delivered by the radiated photons along the tangential direction of the cyclotron orbit would cause the electron's moving speed ${ }^{v}$ to decrease. See the Figure 2.

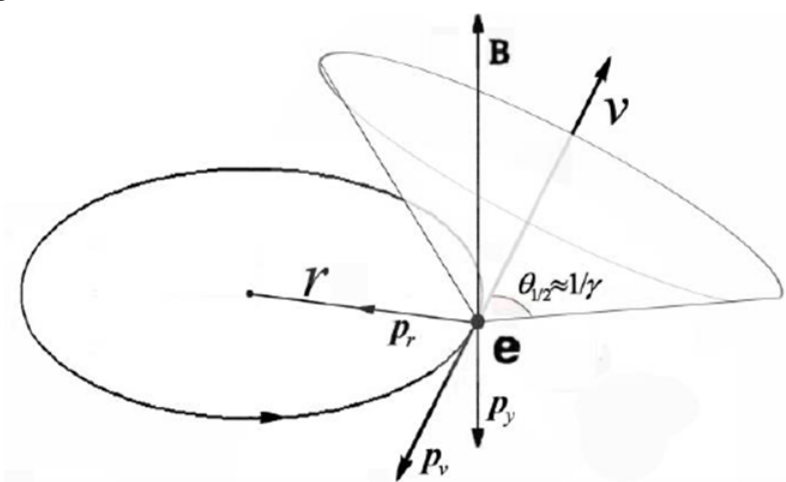

Figure $2 \mathrm{~A}$ schematic diagram of the momentum delivered by a radiated photon to the single electron.

Table I The single electron's movement data in $B=I(T)$ magnetic field

\begin{tabular}{llll}
\hline point & $f_{r}\left(G_{Z}\right)$ & $v(m / s)$ & $r(m m)$ \\
\hline a & 24.7823 & $0.4649832 c$ & 0.895234 \\
b & 24.7868 & $0.46488 I \mathrm{Ic}$ & 0.894983 \\
c & 24.7845 & $0.4648335 \mathrm{c}$ & 0.894866 \\
d & $24.786 \mathrm{I}$ & $0.464746 \mathrm{c}$ & 0.894599
\end{tabular}

In the Figure 2, when radiations occurred, every one of the photons would deliver a momentum $P_{L}$ to the single electron in the form of impulse, which had the same size with the momentum of the photon itself, but the opposite direction. The momentum $P_{L}$ was able to be decomposed into three vectors of the $p_{v}, p_{r}$ and $p_{y}$ The momentum $p_{v}$ was along the tangent direction of the cyclotron orbit, opposite to the direction of the single electron's moving speed $v$ and resulting in a gradual decrease of its moving speed $v$. The momentum $p_{r}$ was along the direction of the orbit radius $r$, and the momentum $p_{y}$ was perpendicular to the plane determined by the $p_{v}$ and $p_{r}$. The momentum $p_{r}$ would cause the single electron to be shifted in the plane determined by the cyclotron orbit. The momentum $p_{y}$ would cause the plane determined by the single electron's cyclotron orbit to be tilted. The two $\left(p_{r}\right.$ and $\left.p_{y}\right)$ acted together on the single electron, until it touched the inner wall of the vacuum chamber. Yes, a complete process of single electron cyclotron was over.

The momentum $\mathrm{Pv}$ caused the moving speed $\mathrm{v}$ of the single electron to decrease. Therefore, according to formulas (2) and (3), its cyclotron frequency $f_{\gamma}$ would be increased, while the orbit radius $r$ was correspondingly decreased. In terms of the momentum $P_{v}$ when its magnitude changed less, the straight-line segment with a slight inclination from point $\mathrm{b}$ to point $\mathrm{c}$ in the Figure 1, could be used as an example. As shown in the Table 1, during this time period, the single electron's moving speed $v$ decreased slowly from $0.4648811 \mathrm{c}$ to $0.4648335 \mathrm{c}$, causing the orbit radius $r$ also to decrease accordingly from $0.8949832(\mathrm{~mm})$ to $0.8948662(\mathrm{~mm})$. Thereupon, the single electron's cyclotron frequency $f_{\gamma}$ also increased from 24.7838 $(\mathrm{GHz})$ to $24.7845(\mathrm{GHz})$. However, the angle and energy of every one of the radiated photons all had randomness, so the magnitude of momentum $P_{V}$ would also present out sharp jump. For example, in the Figure 1, it jumped from point a to point $\mathrm{b}$, as well as from point $\mathrm{c}$ to point $\mathrm{d}$. As shown in the Table 1, the single electron's moving speed $v$ respectively fell sharply from $0.4649832 \mathrm{c}$ to $0.4648811 \mathrm{c}$, and from $0.4648335 \mathrm{c}$ to $0.4647246 \mathrm{c}$; while causing the orbit radius $\mathrm{r}$ also respectively fell sharply from $0.8952338(\mathrm{~mm})$ to 0.8949832 $(\mathrm{mm})$, and from $0.8948662(\mathrm{~mm})$ to $0.8945988(\mathrm{~mm})$. Thereupon, the single electron's cyclotron frequency $f_{\gamma}$ also jumped respectively from $24.7823(\mathrm{GHz})$ to $24.7838(\mathrm{GHz})$, and from $24.7845(\mathrm{GHz})$ to $24.7861(\mathrm{GHz})$.

In terms of the above straight-line segment with a slight inclination from point $\mathrm{b}$ to point $\mathrm{c}$, the electron storage rings widely in use are based on this principle to build the large-scale scientific facilities. In a storage ring, the magnetic induction intensity $B$ and the curvature radius $r$ both are designed as the calibration values. So according to the formula (3), the moving speed $v$ of every one of the electrons is also the calibration value, while the charge-mass ratio $e / m_{0}$ can only be a constant. Otherwise, if the charge $e$ of each electron cannot follow along with its static mass $m_{0}$ to be changed synchronously, while even if the moving speed $v$ can be remained unchanged, the radius $r$ must also be changed along with the change of the charge-mass ratio $e / m_{0}$, which will strike the inner wall of the tubular vacuum chamber of the storage ring. But the lifetimes of the electron beams in storage rings are generally several hours or more, which can be used as the experimental facts. In other words, we have proved that the chargemass ratio $e / m_{0}$ in the formula (3) is constant. Furthermore, in order to compensate for the gradual decrease of the moving speed ${ }^{V}$ of electrons caused by synchronous electromagnetic radiation, there is also a high-frequency cavity system in the storage ring. The electron beam rotating each rotation, its energy will be moderately replenished in the high-frequency cavity, so as to ensure that its moving speed $v$ follows the rules determined by the formula (3).

\section{Conclusion}

The experiments of Project 8 have been excellent, even though the experimental methods changed a lot of patterns, but the expected goals were still difficult to achieve. So much so that some of the results at your fingertips were also missing. This paper points out that the difficulty faced by the scientists at Project 8 is precisely the uncertainty caused by electromagnetic radiations. The above points are summarized as follows:

The value $c$ of the light speed in vacuum and a particle with zero static mass, both of them do not exit in the reality. As the limit values of the change of things in the reality, they strictly follow the rules determined by Newton's first law of motion.

In the reality, photons are high-speed particles generated by electromagnetic radiation. They must have the characteristics of (static) mass, emery and wave, in order to describe the main body to aim at photons from different angles. After any main body comes in to being electromagnetic radiation, its static mass will inevitably decrease accordingly. 
The charge mass ratio of electrons is physical constant, which is the ratio of its charges to the amount of matter, and is not affected by relativistic effects and electromagnetic radiations. When an electron is rotating, its static mass and amount of the charge will not be lost if there is no electromagnetic radiation. The so called uncertainty will not exist. Therefore, the uncertainty of the moving electrons is caused by random electromagnetic radiation.

In a given magnetic field, the moving speed of a single electron to reduce, will cause its orbit radius to decrease, cyclotron frequency to increase, including the orbital circular plane to be shifted and titled, all of which are caused by random electromagnetic radiation.

Finally, it is pointed out that if the cyclotron frequency of a single electron is measured, and at the same time, its de Broglie wavelength or frequency can also be measured, then its static mass as well as corresponding momentum and energy can be obtained. But even so, the expected goals are still difficult to achieve, because random electromagnetic radiations are always taking away continually the matter composition of the single electron being measured. An electron can be further broken down and the texture of each part of the debris is the same, which is precisely a result of the research obtained by the Project 8 after hovering for several years, and has universality, should be reflect on.

A truth cannot be proved by relying on some so-called perfect experiment, ${ }^{4}$ while can only be in a difficult and embarrassing environment to continually modify the one-sided view to approach it. Just like those large-scale particle-physics colliders, the investment and expenditure are exponentially growing, a universal objective law proven finally, also are just so. Matter can be broken down, hope you don't despise this objective law which has universality. Only to take one example, according to the relation formula of de Broglie's matter wave, you will find that the primary factor causing the spectrum red shift is that photons are further broken down by electromagnetic radiation. Well, whether do you still deem only on the basis of the Doppler Effect that almost all of celestial bodies are moving away from us?

\section{Acknowledgments}

None.

\section{Conflicts of interest}

The author declares there is no conflict of interest.

\section{References}

1. DM Asner. (Project 8 Collaboration).Single-electron detection and spectroscopy via relativistic cyclotron radiation [J]. Physical Review Letters. 2015.

2. Jian Ding, Xiuqin HU. Piercing the Veil of Modern Physics. Basics \& Philosophy [M]. Germany: Lambert Academic Publishing, 2017. p. 1-19.

3. Ding Jian, HU Xiuqin. Piercing the Veil of Modern Physics. Part $1 \&$ Basics. Phys Astron Int J. 2018;2(2):128-134.

4. Ding Jian. Piercing the Veil of Modern Physics. Part 2 \& Philosophy. Phys Astron Int J. 2018;2(2):136-144..

5. Ding Jian, HU Xiuqin. Piercing the Veil of Modern Physics: Part 3 \& Superconductivity [J]. Open Access Journal of Physics. 2019;3(1):26-48. 